\title{
Load Situation Awareness Design for Integration in Multi-Energy System
}

\author{
Cai, Hanmin; You, Shi; Bindner, Henrik W.; Klyapovskiy, Sergey
}

Published in:

Proceedings of IEEE International Conference on Energy Internet

Link to article, DOI:

10.1109/ICEI.2017.15

Publication date:

2017

Document Version

Peer reviewed version

Link back to DTU Orbit

Citation (APA):

Cai, H., You, S., Bindner, H. W., \& Klyapovskiy, S. (2017). Load Situation Awareness Design for Integration in Multi-Energy System. In Proceedings of IEEE International Conference on Energy Internet [7926847] IEEE. https://doi.org/10.1109/ICEI.2017.15

\section{General rights}

Copyright and moral rights for the publications made accessible in the public portal are retained by the authors and/or other copyright owners and it is a condition of accessing publications that users recognise and abide by the legal requirements associated with these rights.

- Users may download and print one copy of any publication from the public portal for the purpose of private study or research.

- You may not further distribute the material or use it for any profit-making activity or commercial gain

- You may freely distribute the URL identifying the publication in the public portal 


\title{
Load Situation Awareness Design for Integration in Multi-Energy System
}

\author{
Hanmin Cai, Shi You, Henrik W. Bindner, Sergey Klyapovskiy \\ Department of Electrical engineering \\ Technical University of Denmark \\ Elektrovej, 2800 Kgs. Lyngby, Denmark \\ Email: hacai@elektro.dtu.dk
}

\begin{abstract}
Renewable Energy Sources (RESs) have been penetrating in power system at a staggering pace in recent years. Their intermittent nature is, however, posing great threat to system operation. Recently, active load management has been suggested as a tool to counteract these side effects. In multi-energy system, thermal load management will benefit not only electric network but also district heating network. Electric heater will be the main focus of this paper as a common thermal load type. A situation awareness framework of its integration into electric and district heating network will be presented in this paper.
\end{abstract}

\section{INTRODUCTION}

The last two decades have witnessed substantial development in renewable energy industry and worldwide adoption of green technology, in reaction to energy safety and climate change concerns. Nonetheless, the increasing proliferation of intermittent energy sources like Photovoltaics (PV) installation and wind farms are posing more threat on current power system stability than ever. Instead of prohibitive network reinforcement to increase capability of accommodating volatile energy, researchers have been looking at alternatives such as enabling energy management from the demand side, which is often denoted as Demand Side Management (DSM) in literatures [1]. DSM has been implemented by various utilities mainly for peak shaving [2]. Recent researches have been focusing on DSM's potential to provide ancillary services [3]. Rather than investigating load aggregation for a specific ancillary service, this paper focuses on designing SituationAware (SA) load responding in different situation of district heating $(\mathrm{DH})$ and electric network.

Compared to active research on load aggregation to provide electric network services, relative less research has been going on to investigate how load can assist other networks. The latter will be discussed in this paper under the context of electric and heat multi-energy system. Typical DH system has CHPs as heat sources, equipped with large Heat Pumps (HP), gas boilers and significant heat storage as energy buffer. Gas boilers are dispatched when large HPs fall short of demand. Small CHPs are mostly on-off controlled to produce power when price is high with heat being side product. At the same time, District Heating Operator, as heat supplier, must maintain sufficient heat level to deliver contracted heat when required. In this sense, DH network operation is linked to electricity spot market such as Nord Pool, considering also the fact that electricity demand and thereby prices are also higher during winter time. While CHP depending its size may not be flexible enough to follow fluctuating Renewable Energy Sources (RES) output for up and down regulation, large HPs at plant site can make use of excess power in electric network for heat supply thus reduce gas boilers usage and balance electric network. Hence, CHP serves as a very good link between these two networks at the supply side. Whereas electric heater can function as a coupling of two network operation at the consumer side, especially for countries like Denmark where district heating and local electric heater are often combined as an integrated heating solution to end users. With the purpose of reducing heat loss in DH pipes and lower return water temperature, Low Temperature District Heating (LTDH) has been investigated recently. By reducing the temperature at demand side, the amount of heat to be delivered is reduced. Consequently, temperature needs to be boosted at consumer side by using small-scale HPs or electric heaters to meet end-users heat demand. Usually equipped with heat storage, electric heaters' flexibility has been exploited. For example, heat storage can help to make the most of volatile electricity price by heating when price is low and delay when it is high. Load responding to electricity price must receive external price signal and when communication channel is down, methods such as machine learning must be found such that load functions as communicating link is work.

In this paper, SA load design framework will be illustrated for electric heaters, in that they contribute to a large portion of total energy consumption and their slow dynamics allow frequent interruption without compromising of their original functions significantly. The latter is the source of load's potential to provide services for $\mathrm{DH}$ and electric network. Section II starts with describing the interactions among stakeholders and thus provide a common understanding of multienergy system, on top of which SA framework will be built. Section III will follow by introducing the concept of situation awareness, situation classification and services prioritization. A Finite State Machine (FSM) will be proposed to describe load providing different services subject to situation changes in IV, where controller structure is also discussed. Potential side effect of load group situation awareness will be discussed in section V. Finally, section VI will conclude the paper and some outlook are provided. 
To investigate SA load's integration into future energy system, it is indispensable to describe the stakeholders that load will interact with and their mutual relations. The context is described in figure 1, it consists of Transmission System Operators (TSOs), Distribution System Operators (DSOs), DHOs, Market, Aggregators and Distributed Energy Resources (DERs). With DER refer to electric heater in this paper. Figure 1 builds on Danish context and may vary in other countries.

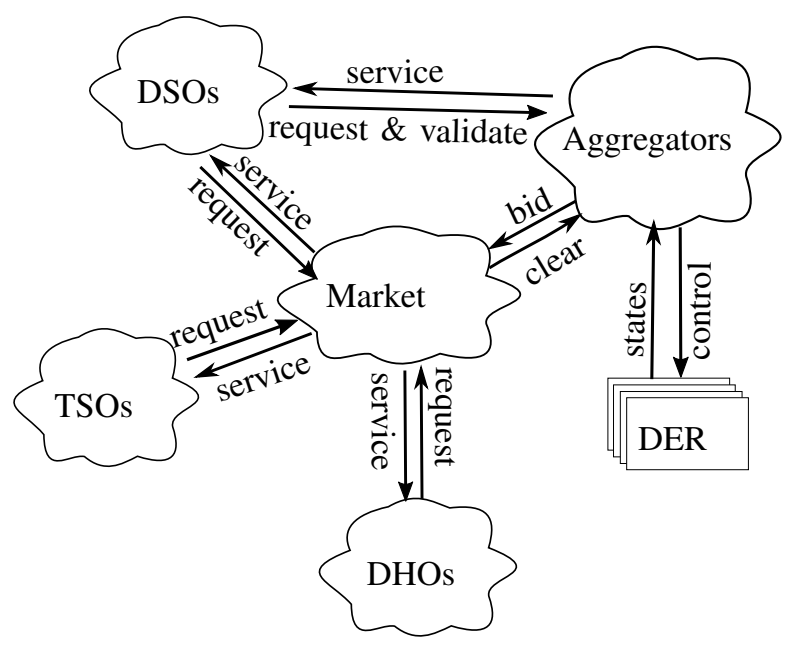

Fig. 1: Stakeholders in electric and heat network

Aggregators considered in this paper refer to those who manage several portfolios composed of thermal loads in distribution network. In order to operate the whole system efficiently, a liberalized market is regarded as the joint between TSOs, DSOs, DHOs and Aggregators. With TSOs, DSOs and DHOs requesting a particular service, Aggregators bid their services and best offer will be accepted by Operators with Market clearing the transactions. Although TSOs may request frequency regulation service from Aggregators, it will be DSOs responsible for managing and validation since consumers are directly connected to them. For example, frequency regulation is mainly requested by TSOs to balance power production and consumption, which requires aggregating large amount of distributed loads. In this sense, DSOs must be involved and evaluate Aggregators' proposal since low voltage network is relatively weak and potential load peak will cause grid congestion. Any compromise in grid reliability will end up with DSOs paying fine because of failure to deliver electricity. Upon receiving service request from the Market, Aggregators design their products by considering the measurement or estimates of states and capability in their resource pool. Although both Aggregators and DSOs have to monitor the response from resource pool, Aggregators will take more responsibility to justify their contribution in order to receive correct compensation.

\section{A. Definition}

Situation awareness originally refers to military flight pilots' crucial quality of combating in changing environment and performing their functions effectively and safely [4]. It reflects the capability of receiving information, analyzing and responding accordingly to certain situation. This capability is illustrated in figure 2 for SA load. This section will first categorize situations that load may encounter, followed by service corresponding to a specific situation and concluded by service prioritization.

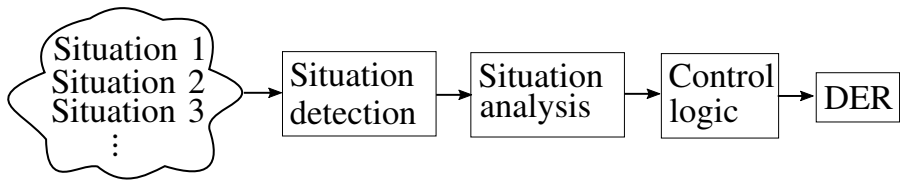

Fig. 2: DER situation awareness illustration

\section{B. Situation categories}

1) Normal situation. In this base scenario, load only has to take care of users' comfort as it is designed for. As for thermal loads, maintaining the temperature inside comfort zone will be the operation logic in this Situation.

2) System recovering from blackout. When network recovers from outage, Electric heaters tend to switch on all together and bring the temperature back into comfort zone. These synchronized loads will generate a huge unexpected load peak.

3) System frequency excursion. Within UCTE, system frequency must be maintained close to $50 \mathrm{~Hz}$, with a maximum fluctuation of $\pm 0.5 \mathrm{~Hz}$ according to ENTSO$\mathrm{E}$ handbook. With increasing penetration of intermittent volatile renewable generations, frequency excursion has been more frequent and wider. Generally, a range of $\pm 10 \mathrm{mHz}$ is implemented to as deadband [5]. Hence this situation can be described as $49.5 \mathrm{~Hz} \leq f \leq$ $49,99 \mathrm{~Hz}$ or $50.01 \mathrm{~Hz} \leq f \leq 50.5 \mathrm{~Hz}$.

4) Feeder voltage deviation. Without the increased Photovoltaique (PV) generation and Electric vehicle (EV) distributed along the distribution line, voltage congestion problem are expected to get worse. Similar to frequency regulation, voltage are regulated in three levels with the acceptable deviation of $0.05 \mathrm{pu}$. The situation can be described as $U \leq 0.95$ pu or $U \geq 1.05$ pu.

5) Volatile electricity market price. With liberalized electricity trading market and highly volatile renewable generation, we here in Denmark can already observe significant price fluctuations. When it is volatile enough, loads are expected to arbitrage electricity by making use of local storage in order to achieve monetary gaining or saving. For example, electric heaters store energy in tank water when price is low and avoid consuming electricity by using stored energy when it is high.

6) District heating operation special schedule. CHPs generally stopped for maintenance for few days, during which 
storage tanks are emptied. Thus no heat will be supplied to customer side. This is usually done by prior notice to customers and scheduling maintenance to times when heat demand is low, such as summer period. In this situation, electric heaters will take over the responsibility to supply heat demand.

7) CHP flexible generation and gas boiler usage reduction. As mentioned before, district heating heat sources CHPs are participating in electricity trading market. Arbitraging electricity while maintaining heat supply level will require electric heaters' assistance. To reduce gas boiler usage in case of large HPs falling short of demand, electric heaters' can be used to help reduce peak heat demand.

8) Emergency load shedding. When system is so stressed that frequency dipped down to its limit and existing reserves are not sufficient to bring it back to normal operating range, load shedding will serve as the last resort to protect our system. Traditionally, DSOs shed all the loads in its region without differentiating. Here, we assume load are differentiable and they switch off voluntarily to allow life-related facilities like hospital to continue operation. This situation is described as $f \leq 49.5 \mathrm{~Hz}$

\section{Service categories}

Possible situations have been defined in section III-B and it is assumed that they are collectively exhaustive to describe the situations that a load may encounter. In this section, load's reactions to aforementioned situations will be discussed in details.

1) Cold Load Pickup Reduction (CLPR). This service aims at reducing the cold load pickup problem by loads detecting if they just recover from outage and reacting in a collectively sensible manner. Several possible options for detection are provided as following:

a) Load receive explicit signal from DSO regarding an occurred outage.

b) Without external signal from DSO, loads can also keep track of the power from mains. The information that there was no power from mains is sufficient to detect outage occurrence. This function will require small battery and memory to $\log$ the information when outage strikes.

c) When considering heat pump, even standby mode requires some minimum power. Hence, load controller is aware of outage by just reading previous power consumption.

Once load detects occurred outage, it will delay the startup for a random period and can be formulated as:

$$
\begin{aligned}
t_{\text {on }}^{i} & =t_{\text {on }}^{i}+\Delta t^{i} \\
\Delta t^{i} & \sim U(0, b)
\end{aligned}
$$

where $\Delta t^{i}$ is the uniformly distributed delay implemented on load $i$. Parameter $b$ is chosen such that the peak power $\left|\sum_{i \in L} P_{i, t}\right|_{\max } \leq P_{\text {normal }}$. Parameter $b$ can be either written in load controller memory as a factory setting or broadcast by DSOs as external control signal. The formulation above applies to load with only ON/OFF mode. Heat pump, for example, will operate in a continuous power reference. Thus the formulation will need to adapt to control the power ramp rate.

2) Fall Back operation (FB). When load are completely isolated due to loss of external signal and local measurements or frequency and voltage stay within normal range, load will operate on fall back mode meaning it only has to take care of users' comfort as it is designed for, i.e. maintaining the temperature inside comfort zone will be thermal load's reaction to this situation. Typical thermal load dynamics can be described by equation 2 [6]. This serves as electric heater basic function.

$$
\begin{aligned}
& \theta_{k+1}^{i}=a^{i} \theta_{k}^{i}+\left(1-a^{i}\right)\left(\theta_{a, k}^{i}-\eta^{i} P_{i n}^{i}\right)+\epsilon_{k}^{i} \\
& \theta_{\text {down }} \leq \theta_{k+1}^{i} \leq \theta_{\text {up }}, \forall k \in R, i \in L
\end{aligned}
$$

where $\theta_{k}^{i}$ denotes $i$ th load temperature in time step $k, P_{i n}^{i}$ as input power, $\eta^{i}$ as performance coefficient, $\theta_{\text {up }}, \theta_{\text {down }}$ as up down temperature limits and stochastic residual $\epsilon_{k}^{i}$ to reflect the real world.

3) Frequency Regulation (FR). Traditionally, this service is structured in three level depending on response time and size of reserve. Within UCTE, these three levels are usually denoted as primary, secondary and tertiary control reserve [5]. Primary control is mostly provided by droop controller on central generator responding to stop frequency excursion within seconds before secondary control kicks in and release primary reserve bringing frequency back to nominal value. Tertiary reserve can take up to one hour to help energy dispatch in a more economical way. Load aggregation to provide ancillary services are investigated in [7], [1] and [8] for primary, secondary and tertiary control reserve respectively. Autonomous frequency regulation load analyzed in [9] is suggested as FR service in the paper.

4) Voltage Regulation (VR). Load aggregation has the potential to mitigate network congestion problem [10]. By controlling load power consumption, voltage control can be achieved at distribution network where $R / X$ ratio is significant. However, the position of loads in the network contribute differently to addressing network congestion problem. Also noted that the further load connected down along distribution line, the more voltage sensitivity to load power injection, thus will need to contribute more in voltage regulation events. Thus brings up the question of fairness for the customer.

5) Price-sensitive load control.

a) Real Time Price Load control (PLC). When communication channel is open load can receive real time price signal, which represents the degree of system stress. Real time price will have the ability to smooth out the load peaks by avoiding the peak 
price and shifting to low price period with low system stress. With this method, peak shaving can be achieved.

b) Pseudo Price Load Control (PPLC). This service as off-line mode of previous PLC service, when price signal is lost due to communication failure. In this case load will rely on pseudo price signal. One way to obtain is by factory setting, in which a generic average price curve is saved in controller's memory. Another way is recreate a price curve by considering the historic price data.

6) District Heating Operation Special Schedule (DHOSS). As mentioned in section III-C, CHP maintenance will cut customer heat supply and electric heater assistance in delivering heat demand is reasonable. As maintenance schedule is usually planned months ahead, thus broadcast this information to all electric heaters for them to store sufficient heat to deliver in maintenance period is feasible.

7) Fuel shifting (FS). Similar to DHOSS, DHOs require electric heater to help guarantee end user comfort under the condition that CHPs are arbitraging in electricity market or simply want to reduce peak demand and thus less gas boiler usage. This can also be achieved by storing sufficient heat according to prior notice from DHOs.

8) Emergency load shedding (OFF). When power system is at limit, load can volunteer to shut down in exchange of economical benefits, contribute to save the system from blackout or reduce severity in return of faster system recovery.

\section{Service priority}

Unlike the conventional dispatch-able generators used for ancillary services, loads considered for this process have their main functionality as delivering energy service to end customer. Unless the system is close to collapse, in which case load shedding is implemented regardless the energy services. Provide demand response to distribution network helps DSOs defer or eliminate grid enforcement investment. Current ancillary services are traded and cleared at the market platform such as Nordpool, the augmented ancillary service capacity should also be compensated considering the reduced investment in DSOs side.

This section mainly discuss a criteria to evaluate control benefit with respect to consumer and power system, or put it another way the priorities of services. Total benefit is defined as:

$$
S:=\sum_{i=1}^{m} w_{i} x_{i}+\sum_{j=1}^{n} w_{j} x_{j}
$$

Where $w_{i}, w_{j}$ denotes the value of $i$ th customer service and $j$ th power system service respectively, control variable $x_{i}, x_{j} \in\{0,1\}$ are binary to denotes a certain service is chosen or not. Additionally, $\sum_{i}^{m} w_{i}=1$ and $\sum_{j}^{n} w_{j}=1$ are required to make services comparable. $w_{i}$ reflects consumer's comfort and monetary gaining or saving, whereas $w_{j}$ reflects the loss in system if this service is not provided or the cost to purchase in the market. Being generic enough, this formulation covers the control target and optimization variable without being limited to a specific type of load. The challenge is, however, how to quantify the value of each service and evaluating $w_{i}$ and $w_{j}$ before formulation 3 can be used in designing controller. To the best of authors' knowledge, explicit numerical values of $w_{i}$ and $w_{j}$ that are generic enough can not be found. Nonetheless, priority order has been defined according to authors' experience and knowledge about their importance and is illustrated in the diagram depicted in figure 4. Alternatively, authors suggest external signal received from aggregators and system operators have the capability to define the priority of services. This option will, however, not be covered in details here.

\section{ADAPTIVE CONTROL STRATEGY}

Since control target varies in different situation, it has to adapt its control strategy as well. To the authors' best knowledge, there is no generic control strategy that applied in every situations.

\section{A. External control signal}

1) Explicit power reference

2) Broadcast signal with load react in stochastic manner

3) Volatile price signal that load react by its greedy nature

As shown in figure 3, device control signal can further classified as discrete and continuous:

1) Discrete control. Devices like water heater has only ON/OFF control mode and generally turn on when temperature fall below a given threshold without extra control.

2) Continuous control. Compressor inside heat pump can operate at continuous power reference, although they run at static power reference to achieve best designed performance. However, continuous control must respect the power rating and ramping rate. Violating either will end up with poor control result by deviating from the power reference. Also standby mode of the devices generally consumes a low level of power.
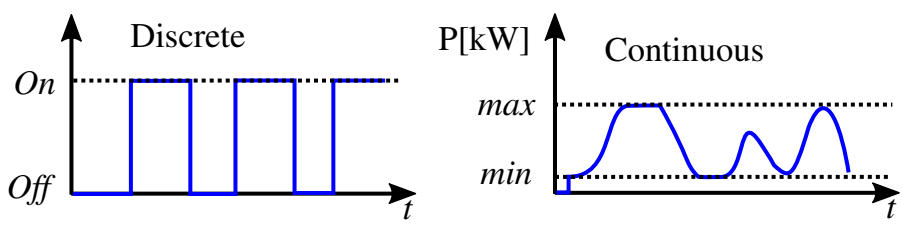

Fig. 3: Discrete and continuous control signal

\section{B. Finite State Machine}

With section III-C listing various services that load can provide in different situations, this section propose to combine these services using Finite State Machine method and result is presented in figure 4 . Load change from one state to another 


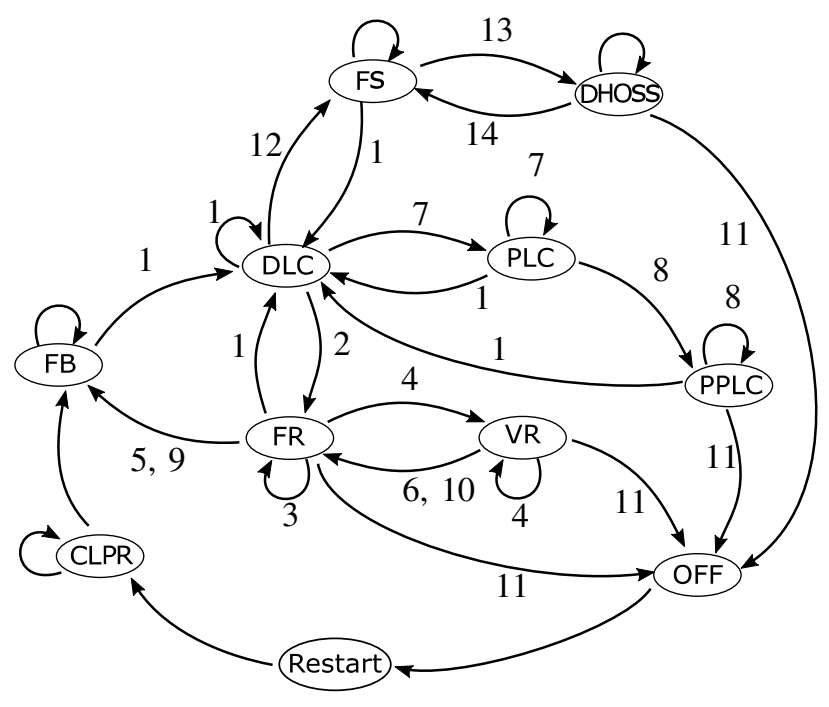

Fig. 4: Finite State Machine

based on events, external signal and local measurements. The transitions between states subject to the following conditions.

1) explicit external control signal received,

2) explicit external control signal lost or received command to switch to FR service,

3) frequency measurement deviates from normal operating range $49.5 \mathrm{~Hz} \leq f \leq 49,99 \mathrm{~Hz}$ or $50.01 \mathrm{~Hz} \leq f \leq$ $50.5 \mathrm{~Hz}$,

4) voltage measurement deviates from normal operating range $U \leq 0.95 \mathrm{pu}$ or $U \geq 1.05 \mathrm{pu}$,

5) local frequency measurement lost,

6) local voltage measurement lost,

7) real time price signal received or received command to switch to PLC service,

8) real time price signal lost,

9) power system frequency back to normal range $49,99 \mathrm{~Hz} \leq f \leq 50.01 \mathrm{~Hz}$,

10) connected distribution line voltage back to normal range $0.95 \mathrm{pu} \leq U \leq 1.05 \mathrm{pu}$,

11) power system frequency at critical moment $f \leq$ $49.5 \mathrm{~Hz}$

12) DHOs broadcast fuel shifting signal or received command to switch to FS service,

13) DHOs broadcast maintenance schedule,

14) Maintenance period finished.

\section{Implementation}

1) Load controller structure: Figure 5 depicts the components in load controller, reflecting load's capability to receive external information, assessing environment, reacting according to predefined state transition diagram and necessary memory to store historical data.

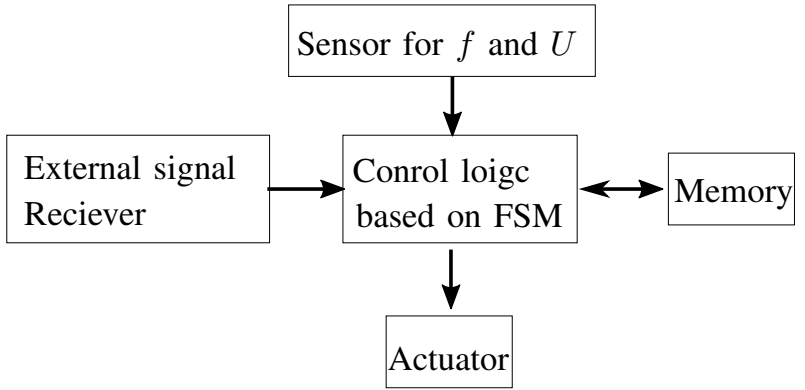

Fig. 5: Load controller structure

2) Additional setting: Signal sources are as following:

\begin{tabular}{l|l|l|l}
\hline Service & Signal source & Offline mode & Offline Sol. \\
\hline CLPR & DSOs & yes & no \\
\hline FB & no & no & no \\
\hline FR & $\begin{array}{l}f \text { local mea- } \\
\text { surement }\end{array}$ & on & on \\
\hline DLC & $\begin{array}{l}\text { external } \\
\text { Aggregators, } \\
\text { DSOs, DHOs }\end{array}$ & no & no \\
\hline PLC & $\begin{array}{l}\text { external } \\
\text { Aggregators, } \\
\text { DSOs }\end{array}$ & yes & $\begin{array}{l}\text { using machine } \\
\text { learning or by } \\
\text { factory setting }\end{array}$ \\
\hline VR & DSOs & no & no \\
\hline DHOSS & DHOs & yes & $\begin{array}{l}\text { historical data, } \\
\text { stored sched- } \\
\text { ule }\end{array}$ \\
\hline FS & DHOs & no & no \\
\hline
\end{tabular}

V. IMPACT OF AUTONOMOUS LOAD GROUP

Previous sections classify the network services and describe how load must react in different scenarios by designing the finite state machine as shown in figure 4. By far, all aforementioned work focuses on a single load and it is control, which mainly affects at the consumers' level. However, network operator concerns more about the effect when there are many loads designed under aforementioned framework in the network. One potential problem could occur arise is that these loads synchronize with each other by coincidence. One possible adverse effect is load synchronization. Load diversity is essential to solve this problem. Probabilistic approach is one way to restore the diversity. When loads are aggregated to provide ancillary services, they work outside of their daily routine. Water heaters, for example, might be controller to delay their power for some period and water inside tank becomes cool such that when aggregator release the control, this pool of water heaters will start up at the same time to bring the water temperature back to normal value.

\section{CONCLUSION AND DISCUSSION}

This paper proposes a framework for designing load situation awareness, which is equipped with situation awareness to assist electric and district heating network operation. Lack of manufactures' intention to produce SA loads and consumers' 
to adopt are preventing demand response from being massively deployed. This paper starts from describing the context where SA load will be integrated. It is followed with categorizing situations and corresponding network services loads may provide. A finite state machine diagram is presented to for design load situation awareness.

Although Demand Response (DR) attracts many researchers with excellent researches being done, what hinders the implementation is lack of active load in the market and underlying Information and Communications Technology (ICT) infrastructure. Trying to change the situation, researchers have been investigating the monetary gaining or saving in load aggregation program, to create a business model and motivate manufacturers to design SA products and consumers to adopt. Instead, in this paper authors suggest demand response be regarded as compulsory feature for every load. Furthermore, this functionality must be certified and labeled on the product. A concept resembles energy saving labeling system, under which manufacturers are obliged to develop energy saving technology as market entry criteria. Although policy design is out of the scope of this paper, the authors believe that additional costs will be negligible once the concept is researched and developed massively. Eventually, there will be more SA load and create huge resource pool to be dispatched.

On the other hand, although IT security is outside the scope of this discussion, it will need to be considered at the beginning of controller design. The more communication interfaces loads are equipped with, the more risk they are exposed to hackers.

\section{REFERENCES}

[1] D. S. Callaway, "Tapping the energy storage potential in electric loads to deliver load following and regulation, with application to wind energy," Energy Conversion and Management, vol. 50, no. 5, pp. 1389-1400, 2009.

[2] P. Cappers, C. Goldman, and D. Kathan, "Demand response in us electricity markets: Empirical evidence," Energy, vol. 35, no. 4, pp. 15261535,2010

[3] S. Koch, Demand response methods for ancillary services and renewable energy integration in electric power systems. $\mathrm{PhD}$ thesis, Citeseer, 2012.

[4] M. R. Endsley, "Toward a theory of situation awareness in dynamic systems," Human Factors: The Journal of the Human Factors and Ergonomics Society, vol. 37, no. 1, pp. 32-64, 1995.

[5] Y. G. Rebours, S. Member, D. S. Kirschen, and M. Trotignon, "A Survey of Frequency and Voltage Control Ancillary Services Part I : Technical Features," vol. 22, no. 1, pp. 350-357, 2007.

[6] J. L. Mathieu, M. Kamgarpour, J. Lygeros, G. Andersson, and D. S. Callaway, "Arbitraging intraday wholesale energy market prices with aggregations of thermostatic loads," IEEE Transactions on Power Systems, vol. 30, no. 2, pp. 763-772, 2015.

[7] E. Vrettos, C. Ziras, and G. Andersson, "Fast and reliable primary frequency reserves from refrigerators with decentralized stochastic control," IEEE Transactions on Power Systems, 2016.

[8] H. Cai, A. Hutter, E. Olivero, P. Roduit, and P. Ferrez, "Load shifting for tertiary control power provision," in 2015 IEEE 5th International Conference on Power Engineering, Energy and Electrical Drives (POWERENG), pp. 469-475, IEEE, 2015.

[9] P. J. Douglass, J. Østergaard, and P. Nyeng, Integrating autonomous load controllers in power systems. PhD thesis, Energinet. dkEnerginet. dk, 2015.

[10] K. Christakou, D.-C. Tomozei, J.-Y. Le Boudec, and M. Paolone, "Gecn: Primary voltage control for active distribution networks via real-time demand-response," IEEE Transactions on Smart Grid, vol. 5, no. 2, pp. 622-631, 2014. 